\title{
On the spectral sequence of the Swiss-cheese operad
}

\author{
EDUARDO HOEFEL \\ MURIEL LIVERNET
}

\begin{abstract}
We prove that the homology of the Swiss-cheese operad is a Koszul operad. As a consequence, we obtain that the spectral sequence associated to the stratification of the compactification of points on the upper half plane collapses at the second stage, proving a conjecture by A Voronov in [17]. However, we prove that the operad obtained at the second stage differs from the homology of the Swiss-cheese operad.
\end{abstract}

18G55, 18D50

\section{Introduction}

An operad $\mathcal{O}$ is called differentiable when it is defined on the symmetric monoidal category of differentiable manifolds. If each $\mathcal{O}(n)$ is a manifold with corners whose connected boundary components are cartesian products of $\mathcal{O}(k)$ (with $k<n$ ) and the operad structure is given by the inclusion map of the boundary strata, then the operad is called stratified. To every stratified operad are associated dg-operads given by the spectral sequence induced by a natural filtration on its singular chain complex: the boundary strata filtration. Since that filtration is given by the codimension of the boundary components, it is finite and hence converges to the homology $H_{*}(\mathcal{O})$ at some finite stage. One would naturally wonder whether the spectral sequence degenerates and if the operad structure on the $E^{\infty}$-term is isomorphic to the operad structure on $H_{*}(\mathcal{O})$.

In the present paper we study the spectral sequence of a stratified operad, given by Kontsevich's compactification [14], which is homotopically equivalent to the Swisscheese operad $\mathcal{S C}$. Among the related algebraic structures are Kajiura and Stasheff's OCHA [10; 12], Leibniz pairs (see Flato, Gerstenhaber and Voronov [3]) and extensions of those considered by Dolgushev [2]. The relation between OCHAS, Leibniz pairs and the Swiss-cheese operad has been carefully studied by the authors in [9], where the $0^{\text {th }}$ homology of the Swiss-cheese operad $\mathcal{S C}$ was related to the first row of the spectral sequence associated to the Kontsevich compactification. One of the purposes of [9] was to prove an $\mathcal{S C}$ analogue of the following fact concerning the little disks operad $\mathcal{D}_{2}$. 
Proposition The $0^{\text {th }}$ homology of the operad $\mathcal{D}_{2}$ is Koszul dual to a suspension of the top homology.

In the case of little disks, the $0^{\text {th }}$ homology is the operad $\mathcal{C}$ om and the top homology is a desuspension of the operad $\mathcal{L}$ ie. In fact, the above Proposition is a consequence of a theorem, proved by Getzler and Jones, according to which the Gerstenhaber operad $H_{*}\left(\mathcal{D}_{2}\right)$ is, up to suspension, a self-dual Koszul operad.

We proved in [9] that the $0^{\text {th }}$ homology of $\mathcal{S C}$ is a Koszul quadratic-linear operad, and that its Koszul dual $H_{0}(\mathcal{S C})^{\text {! }}$, which is a dg-operad, has for homology a suspension of the top homology. Note that in the context of $\mathcal{S C}$, the top homology does not form an operad, so by top homology we mean the smallest operad containing the top degrees generators.

The little disks operad $\mathcal{D}_{2}$ is not stratified. However, by considering the real FultonMacPherson compactification of the moduli space of points in the complex plane, we get a homotopically equivalent stratified operad sometimes denoted $\mathcal{F}_{2}$ (see Salvatore [16]). The same compactification procedure can be applied to the Swiss-cheese operad $\mathcal{S C}$ (the homotopy equivalent stratified operad obtained is sometimes denoted $\mathcal{H}_{2}$ ). So, by passing to a homotopy equivalent operad, we can assume that both little disks and Swiss-cheese operads are stratified. Furthermore, two homotopy equivalent operads give isomorphic homology operads. Hence, to avoid cumbersome notation we will work with the stratified versions of little disks and Swiss-cheese, while keeping the notation: $\mathcal{D}_{2}$ and $\mathcal{S C}$.

The main result of this paper is Theorem 4.2.2, where we prove the conjecture by A Voronov in [17] stating that the spectral sequence $E(S C)$ of the Swiss-cheese operad collapses at the second stage. This is done by proving that the homology of the Swisscheese operad is a quadratic-linear Koszul operad in the sense of Galvez-Carillo, Tonks and Vallette in [5]. The same result is true for the homology of $\mathcal{S C}^{\text {vor }}$, a variant of $\mathcal{S C}$. The relation (modulo (de)suspension) between $E^{1}(S C)$ and the cobar construction of the cohomology cooperad $H^{*}(\mathcal{S C})$ is well known [2], but in our setting it is slightly different, so a proof is given in Lemma 4.1.1. We compute in Proposition 4.2.1 the operad structure on $E^{2}(S C)=E^{\infty}(S C)$. To sum up we get the following.

Algebras over $H_{*}(S C)$ are triples $(G, A, f)$ where $G$ is a Gerstenhaber algebra, $A$ is an associative algebra and $f: G \rightarrow A$ is a central map such that $f\left(g g^{\prime}\right)=f(g) f\left(g^{\prime}\right)$, whereas algebras over $E^{\infty}(S C)$ are triples $(G, A, f)$, where $G$ is a Gerstenhaber algebra, $A$ is an associative algebra and $f: G \rightarrow A$ is a central map such that $f\left(g g^{\prime}\right)=0$.

We finally prove in Proposition 4.3.1 that the two operads are not isomorphic as operads, though the $\mathbb{S}$-module structures are the same. 
Note that this case differs from the little disks case: Getzler and Jones [6] have proven that the spectral sequence associated to the stratification of $\mathcal{D}_{2}$ collapses at the second stage and that $E^{2}\left(\mathcal{D}_{2}\right)=\Lambda^{-1}\left(H_{*}\left(\mathcal{D}_{2}\right)^{!}\right)$is isomorphic to $\mathbf{e}_{2}=H_{*}\left(\mathcal{D}_{2}\right)$.

The plan of the paper is the following. Section 2 is devoted to preliminaries and notation. Section 3 is devoted to the homology of the operads $\mathcal{S C}^{\text {vor }}$ and $\mathcal{S C}$. As in [9], in order to understand the structure of the operad $\mathbf{s c}=H_{*}(\mathcal{S C})$, it is necessary to first understand the operad $\mathcal{S C}^{\text {vor }}$, another version of the Swiss-cheese operad, whose homology is quadratic. The end of Section 3 is devoted to the structure of $H_{*}\left(\mathbf{s c}^{!}\right)$. We use the techniques of distributive laws, as well as the results obtained in [9]. Section 4 concentrates on the spectral sequence of $\mathcal{S C}$.

Acknowledgements This collaboration of the authors is funded by the MathAmSud program "OPECSHA" coordinated by M Ronco, by Fundação Araucária (Brazil) grant FA 490/6032 and by the réseau Franco-brésilien de mathématiques.

\section{Preliminaries}

\subsection{On differential graded vector spaces}

We work on a ground field $k$ of characteristic 0 . The category dgvs is the category of lower graded $k$-vector spaces together with a differential of degree -1 . Objects in dgvs are called for short dgvs. The degree of $x \in V$, where $V$ is a dgvs is denoted by $|x|$. We say that a dgvs $V$ is finite dimensional if for each $n$, the vector space $V_{n}$ is finite dimensional.

The vector space $\operatorname{hom}_{k}(V, W)$ denotes the $k$-linear morphisms between two vector spaces $V$ and $W$. When $V$ and $W$ are objects in dgvs, then we have that the differential graded vector space of maps from $V$ to $W$ is $\bigoplus_{i \in \mathbb{Z}} \operatorname{Hom}_{i}(V, W)$, where $\operatorname{Hom}_{i}(V, W)=\prod_{n} \operatorname{hom}_{k}\left(V_{n}, W_{n+i}\right)$ together with the differential $(\partial f)(v)=$ $\mathrm{d}_{W}(f(v))-(-1)^{|f|} f\left(d_{V} v\right)$.

The graded linear dual of $V$ in $\mathbf{d g v s}$ is $V^{*}=\operatorname{Hom}(V, k)$, where $k$ is concentrated in degree 0 with 0 -differential. Consequently we have that $\left(V^{*}\right)_{n}=\left(V_{-n}\right)^{*}$ and $(\partial f)(x)=-(-1)^{n} f\left(d_{V} x\right)$ for any $f \in\left(V^{*}\right)_{n}$ and $x \in V_{-n+1}$. The suspension of a dgvs $V$ is denoted by $s V$ and defined as $(s V)_{n}=V_{n-1}$.

\subsection{On operads, 2-colored operads, cooperads}

2.2.1 On the symmetric group The symmetric group acting on $n$ elements is denoted by $S_{n}$. An element $\sigma \in S_{n}$ will be denoted by its image notation $(\sigma(1) \cdots \sigma(n))$. The trivial representation of $S_{n}$ is denoted by $k$, the signature representation by $\operatorname{sgn}_{n}$ and the regular representation by $k\left[S_{n}\right]$. 
2.2.2 Collections and $\mathbb{S}$-modules In this article, we work with 2 -colored (co)operads, either in the category of spaces, or in dgvs. The colors we consider are denoted by $c$ (for closed) and $o$ (for open). A 2 -collection $\mathcal{P}$ is a family of dgvs,

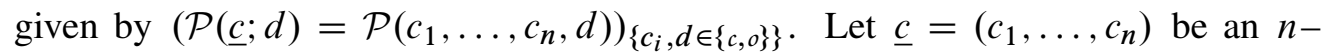
tuple of colors. The symmetric group $S_{n}$ acts on the set of $n$-tuples of colors by $\underline{c} \cdot \sigma=\left(c_{\sigma(1)}, \ldots, c_{\sigma(n)}\right)$. An $\mathbb{S}$-module is a 2 -collection $\mathcal{P}$ endowed with an action of the symmetric groups, sending $\left(x \in \mathcal{P}\left(c_{1}, \ldots, c_{n} ; d\right), \sigma \in S_{n}\right)$ to $x \cdot \sigma \in \mathcal{P}(\underline{c} \cdot \sigma ; d)$.

Note that it is an extension of the usual definition of an $\mathbb{S}$-module, which is a family of dgvs $(\mathcal{Q}(n))_{n \geq 1}$ such that for each $n, \mathcal{Q}(n)$ is a right $S_{n}$-module. We can consider this collection as an $\mathbb{S}$-module, where $\mathcal{Q}(\underline{c} ; d)$ is $\mathcal{Q}(n)$ if for all $i, c_{i}=c$ and $d=c$ and is 0 otherwise.

Given an $\mathbb{S}$-module $M$, we may want to consider some truncation $N$ of it, invariant under the action of the symmetric groups. By definition a sub- $\mathbb{S}-$ module of $N$ is a sub-dgvs invariant under the induced action of the symmetric groups.

2.2.3 Operads The 2 -collection $I$ defined by $I(c ; c)=k, I(o ; o)=k$ and $I(\underline{c} ; d)=0$ elsewhere, plays a special role. Indeed, a 2-colored operad is an $\mathbb{S}$-module together with a unit map $\eta: I \rightarrow \mathcal{P}$ and composition maps

$$
\gamma: \mathcal{P}\left(c_{1}, \ldots, c_{n} ; d\right) \otimes \mathcal{P}\left(\underline{b}^{1} ; c_{1}\right) \otimes \cdots \otimes \mathcal{P}\left(\underline{b}^{n} ; c_{n}\right) \rightarrow \mathcal{P}\left(\underline{b}^{1}, \ldots, \underline{b}^{n} ; d\right),
$$

which are associative, unital and respects the action of the symmetric groups.

We write $f\left(g_{1}, \ldots, g_{n}\right)$ for the image of $f \otimes g_{1} \otimes \cdots \otimes g_{n}$ or $f\left(\mathrm{id}^{\otimes i} \otimes g \otimes \mathrm{id}^{\otimes n-1-i}\right)$ whenever every $g$ except one is the identity. We often use the same notation for $f$ in $\mathcal{P}$ or for $f$ seen as an operation on variables. In that context, we use the Koszul sign convention

$$
(f \otimes g)(\underline{a} \otimes \underline{b})=(-1)^{|a||g|} f(a) \otimes g(b) .
$$

Because of the action of the symmetric groups, one may only consider the spaces

$$
\mathcal{P}(n, m ; d)=\mathcal{P}(\underbrace{c, \ldots, c}_{n \text { times }}, \underbrace{o, \ldots, o}_{m \text { times }} ; d) .
$$

In this paper, we only consider 2 -colored operads such that $\mathcal{P}(0,0 ; x)=0$ and $\mathcal{P}(1,0 ; c)=k=\mathcal{P}(0,1 ; o)$. They are naturally augmented, that is, there is a morphism of operads $\mathcal{P} \rightarrow I$ and $\overline{\mathcal{P}}$ denotes the kernel of this map.

Any operad $\mathcal{P}$ can be considered as a 2 -colored operad with $\mathcal{P}(\underline{c} ; d)=\mathcal{P}(n)$ if for all $i, c_{i}=c$ and $d=c, \mathcal{P}(o ; o)=k$ and $\mathcal{P}(\underline{c} ; d)=0$ otherwise.

In the sequel, we often use the generic terminology of operads for either operads or 2 -colored operads, or operads seen as 2-colored operads. 
2.2.4 Suspension of $\mathbb{S}$-modules and operads The suspension of the $\mathbb{S}$-module $\mathcal{P}$ is

$$
\Lambda \mathcal{P}(n, m ; x)=s^{1-n-m} \mathcal{P}(n, m ; x) \otimes \operatorname{sgn}_{n+m} .
$$

If $\mathcal{P}$ is an operad, then the structure of $\mathcal{P}$-algebra on the pair $\left(V_{c}, V_{o}\right)$ is equivalent to the structure of $\Lambda \mathcal{P}$-algebra on the pair $\left(s V_{c}, s V_{o}\right)$.

The suspension of the $2-$ collection $\mathcal{P}$ with respect to the color $c$ is

$$
\Lambda_{c} \mathcal{P}(n, m ; x)=s^{\delta_{x, c}-n} \mathcal{P}(n, m ; x) \otimes \operatorname{sgn}_{n},
$$

where $\delta$ denotes the Kronecker symbol. If $\mathcal{P}$ is an operad, then the structure of $\mathcal{P}-$ algebra on the pair $\left(V_{c}, V_{o}\right)$ is equivalent to the structure of $\Lambda_{c} \mathcal{P}$-algebra on the pair $\left(s V_{c}, V_{o}\right)$.

2.2.5 Operads defined by generators and relations The free operad generated by an $\mathbb{S}$-module $E$ is denoted by $\mathcal{F}(E)$. It is weight graded by the number $n$ of vertices of the underlying trees and $\mathcal{F}^{(n)}(E)$ denotes the component of weight $n$.

A quadratic operad $\mathcal{F}(E, R)$ is an operad of the form $\mathcal{F}(E) /(R)$, where $E$ is an $\mathbb{S}$-module, $R$ is a sub- $\mathbb{S}$-module of $\mathcal{F}^{(2)}(E)$ and $(R)$ is the ideal generated by $R$. There are analogous notions of cooperads, free cooperads $\mathcal{F}^{c}(E)$ cogenerated by $E$, and of cooperads cogenerated by an $\mathbb{S}$-module $V$ with correlation $R$ denoted by $C(V, R)$.

Describing an operad is equivalent to describing algebras over it. In the text, we say that an operad $\mathcal{P}$ is generated by $E$ with relations $R$ written as

$$
r_{1}=r_{2} .
$$

This notation means that any $\mathcal{P}$-algebra satisfies the relation $(*)$. At the level of operads, this is understood as $R$ contains the element $r_{1}-r_{2}$.

2.2.6 Koszul dual Any quadratic operad $\mathcal{P}=\mathcal{F}(E, R)$ admits a Koszul dual cooperad given by $\mathcal{P i}=C\left(s E, s^{2} R\right)$.

The Koszul dual operad $\mathcal{P}^{!}$of a finite dimensional quadratic operad $\mathcal{P}$ is

$$
\mathcal{P}^{!}:=\left(\Lambda \mathcal{P}^{i}\right)^{*} \text {, or equivalently, } \mathcal{P}^{i}=\left(\Lambda \mathcal{P}^{!}\right)^{*} .
$$

When $\mathcal{P}$ is a binary quadratic operad, we can use the original definition of Ginzburg and Kapranov [7] (see also Loday and Vallette [15, chapter 7]) to compute its Koszul dual operad. Namely, if $\mathcal{P}=\mathcal{F}(E, R)$, then $\mathcal{P}^{!}=\mathcal{F}\left(E^{\vee}, R^{\perp}\right)$, where $E^{\vee}=E^{*} \otimes \operatorname{sgn}_{2}$ and $R^{\perp}$ denotes the orthogonal of $R$ under the pairing $\mathcal{F}^{(2)}(E) \otimes \mathcal{F}^{(2)}\left(E^{\vee}\right) \rightarrow k$. 
2.2.7 Bar and cobar constructions As it is the case for algebras and coalgebras, there is a pair of adjoint functors between operads and cooperads given by the bar and cobar construction. We refer to [6] for a detailed account on this topic. Let us only recall that the cobar construction of a cooperad $\mathcal{C}$ is denoted by $\Omega \mathcal{C}$ and as a (nondifferential) operad, is the free operad $\mathcal{F}\left(s^{-1} \overline{\mathcal{C}}\right)$ where $\overline{\mathcal{C}}$ is the coaugmentation ideal of the cooperad. The differential is computed from the differential of $\mathcal{C}$ and the cooperad structure. The bar construction is denoted $B \mathcal{P}$ and it is defined similarly. When $\mathcal{C}$ is finite dimensional one has

$$
(\Omega \mathcal{C})^{*}=B\left(\mathcal{C}^{*}\right)
$$

\subsection{Two versions of the Swiss-cheese operad}

Here we recall the two definitions for the Swiss-cheese operad we have introduced in [9]. We denote by $\mathcal{D}_{2}$ the little disks operad.

For $m, n \geqslant 0$ such that $m+n>0$, let us define $\mathcal{S C}(n, m ; o)$ as the space of those configurations $d \in(2 n+m)$ such that its image in the disk $D^{2}$ is invariant under complex conjugation and exactly $m$ little disks are left fixed by conjugation. A little disk that is fixed by conjugation must be centered at the real line, in this case it is called open. Otherwise, it is called closed. The little disks in $\mathcal{S C}(n, m ; o)$ are labelled according to the following rules.

(i) Open disks have labels in $\{1, \ldots, m\}$ and closed disks have labels in $\{1, \ldots, 2 n\}$.

(ii) Closed disks in the upper half plane have labels in $\{1, \ldots, n\}$. If conjugation interchanges the images of two closed disks, their labels must be congruent modulo $n$.

There is an action of $S_{n} \times S_{m}$ on $\mathcal{S C}(n, m ; o)$ extending the action of $S_{n} \times\{e\}$ on pairs of closed disks having modulo $n$ congruent labels and the action of $\{e\} \times S_{m}$ on open disks. Figure 1 illustrates a point in the space $\mathcal{S C}(n, m ; o)$.

The 2-collection $\mathcal{S C}$ is defined as follows. For $m, n \geqslant 0$ with $m+n>0, \mathcal{S C}(n, m ; o)$ is the configuration space defined above and $\mathcal{S C}(0,0 ; o)=\varnothing$. For $n \geqslant 0, \mathcal{S C}(n, 0 ; c)$ is defined as $\mathcal{D}_{2}(n)$ and $\mathcal{S C}(n, m ; c)=\varnothing$ for $m \geqslant 1$. The 2 -colored operad structure in $\mathcal{S C}$ is given, as usual, by insertion of disks.

There is a suboperad $\mathcal{S C}^{\text {vor }}$ of $\mathcal{S C}$ defined by $\mathcal{S C}^{\text {vor }}(n, m ; x)=\mathcal{S C}(n, m ; x)$, if $x=c$ or $m \geqslant 1$ and by $\mathcal{S C}^{\text {vor }}(n, m ; x)=\varnothing$, otherwise. The above definition says that $\mathcal{S C}^{\text {vor }}$ coincides with $\mathcal{S C}$ except for $m=0$ and $x=o$, where $\mathcal{S C}^{\text {vor }}(n, 0, o)=\varnothing$ for any $n \geqslant 0$. The operad $\mathcal{S C}^{\text {vor }}$ is equivalent to the one defined by Voronov in [17], while $\mathcal{S C}$ coincides with the one defined by Kontsevich in [13]. 


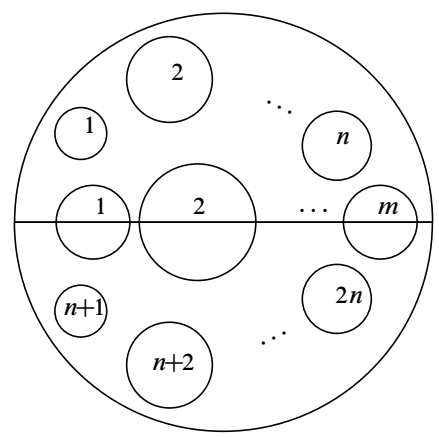

Figure 1: A configuration in $\mathcal{S C}(n, m ; o)$

Notation The homology of $\mathcal{S C}$ is denoted by sc while that of $\mathcal{S C}^{\text {vor }}$ is denoted by $\mathbf{s c}^{\text {vor }}$.

\subsection{Conventions and notation}

2.4.1 Generators In the paper we will have specific generators in the different operads considered, mainly two families of elements. The first is $\left\{f_{2}, g_{2}, e_{0,2}, e_{1,1}, e_{1,0}\right\}$ and the second family is $\left\{\mathfrak{l}_{2}, \mathfrak{c}_{2}, \mathfrak{n}_{0,2}, \mathfrak{n}_{1,1}, \mathfrak{n}_{1,0}\right\}$.

The following array sum up the properties of the elements. The array must be read as follows: $f_{2} \in M(c, c ; c)$ means that it is an operation on two closed variables giving a closed variable; the representation is $k$, that is, $f_{2}$ is a symmetric operation. The degree is 0 .

\begin{tabular}{|c|c|c|c|c|c|}
\hline element & $f_{2}$ & $g_{2}$ & $e_{0,2}$ & $e_{1,1}$ & $e_{1,0}$ \\
color & $M(c, c ; c)$ & $M(c, c ; c)$ & $M(o, o ; o)$ & $M(c, o ; o)$ & $M(c ; o)$ \\
representation & $k$ & $k$ & $k\left[S_{2}\right]$ & $k\left[S_{2}\right]$ in $M(c, o ; o) \oplus M(o, c ; o)$ & $k$ \\
degree & 0 & 1 & 0 & 0 & 0 \\
\hline element & $\mathfrak{l}_{2}$ & $\mathfrak{c}_{2}$ & $\mathfrak{n}_{0,2}$ & $\mathfrak{n}_{1,1}$ & $\mathfrak{n}_{1,0}$ \\
color & $M(c, c ; c)$ & $M(c, c ; c)$ & $M(o, o ; o)$ & $M(c, o ; o)$ & $M(c ; o)$ \\
representation & $\operatorname{sgn}_{2}$ & $\operatorname{sgn}_{2}$ & $k\left[S_{2}\right]$ & $k\left[S_{2}\right]$ in $M(c, o ; o) \oplus M(o, c ; o)$ & $k$ \\
degree & 0 & -1 & 0 & 0 & -1 \\
\hline
\end{tabular}

Given elements $\left\{x_{1}, \ldots, x_{n}\right\}$ with specific colors, representation and degrees, the $\mathbb{S}$ module $\left\langle x_{1}, \ldots, x_{n}\right\rangle$ is the $\mathbb{S}$-module generated by these elements, with the action of the symmetric group indicated by the representation of the elements. For example $\left\langle e_{1,1}\right\rangle$ is the $\mathbb{S}$-module $M$ where $M(c, o ; o)=k e_{1,1}, M(o, c ; o)=k e_{1,1} \cdot(21)$ and is zero elsewhere. 
2.4.2 Notation for operads The operad $\mathcal{G e r}$, whose algebras are Gerstenhaber algebras is the operad $\mathcal{F}\left(E_{\mathcal{G} e r}, R_{\mathcal{G} e r}\right)$ with $E_{\mathcal{G} e r}=\left\langle f_{2}, g_{2}\right\rangle$ and $R_{\mathcal{G} e r}$ is the space of relations given by

$$
\begin{aligned}
f_{2}\left(\mathrm{id} \otimes f_{2}\right) & =f_{2}\left(f_{2} \otimes \mathrm{id}\right), \\
g_{2}\left(g_{2} \otimes \mathrm{id}\right) \cdot((123)+(231)+(312)) & =0 \\
g_{2}\left(\mathrm{id} \otimes f_{2}\right) & =f_{2}\left(g_{2} \otimes \mathrm{id}\right)+f_{2}\left(\mathrm{id} \otimes g_{2}\right) \cdot(213) .
\end{aligned}
$$

The suboperad generated by $f_{2}$ is the operad $\mathcal{C} o m=\mathcal{F}\left(\left\langle f_{2}\right\rangle, R_{\mathcal{C o m}}\right)$ where $R_{\mathcal{C o m}}$ is the first relation. The suboperad generated by $g_{2}$ is the operad $\Lambda^{-1} \mathcal{L} i e$.

The Koszul dual of the operad $\mathcal{G e r}$ is $\mathcal{G e r} !=\Lambda \mathcal{G e r}$ (see eg [6]). It is described as $\mathcal{F}\left(E_{\Lambda \mathcal{G} e r}, R_{\Lambda \mathcal{G} e r}\right)$ with $E_{\Lambda \mathcal{G} e r}=\left\langle\mathfrak{l}_{2}, \mathfrak{c}_{2}\right\rangle$ and $R_{\Lambda \mathcal{G} e r}$ is the space of relations given by

$$
\begin{aligned}
\mathfrak{c}_{2}\left(\mathrm{id} \otimes \mathfrak{c}_{2}\right) & =-\mathfrak{c}_{2}\left(\mathfrak{c}_{2} \otimes \mathrm{id}\right), \\
\mathfrak{l}_{2}\left(\mathfrak{l}_{2} \otimes \mathrm{id}\right) \cdot((123)+(231)+(312)) & =0, \\
\mathfrak{l}_{2}\left(\mathrm{id} \otimes \mathfrak{c}_{2}\right) & =\mathfrak{c}_{2}\left(\mathfrak{l}_{2} \otimes \mathrm{id}\right)+\mathfrak{c}_{2}\left(\mathrm{id} \otimes \mathfrak{l}_{2}\right) \cdot(213) .
\end{aligned}
$$

The suboperad generated by $\mathfrak{l}_{2}$ is the operad $\mathcal{L} i e=\mathcal{F}\left(\left\langle\mathfrak{l}_{2}\right\rangle, R_{\mathcal{L} i e}\right)$, where $R_{\mathcal{L} i e}$ is the second relation. The suboperad generated by $\mathfrak{c}_{2}$ is the operad $\Lambda \mathcal{C}$ om. The operad $\mathcal{A s s}$ is described as $\mathcal{F}\left(\left\langle e_{0,2}\right\rangle, R_{\mathcal{A} s s}\right)$ where $R_{\mathcal{A} s s}$ is the relation $e_{0,2}\left(\mathrm{id} \otimes e_{0,2}\right)=e_{0,2}\left(e_{0,2} \otimes \mathrm{id}\right)$. Note that we also use this notation replacing $e_{0,2}$ by $\mathfrak{n}_{0,2}$.

\section{The homology operads sc ${ }^{\text {vor }}$ and sc}

We prove in this section that the homology operad $\mathbf{s c}^{\text {vor }}$ is a quadratic Koszul operad and that the homology operad sc is a quadratic-linear Koszul operad, extending the results obtained for the $0^{\text {th }}$ homology of $\mathcal{S C}{ }^{\text {vor }}$ and $\mathcal{S C}$ in [9].

\subsection{The operad sc ${ }^{\text {vor }}$ is Koszul}

Recall the following theorem.

Theorem 3.1.1 (A Voronov [17]) An algebra over $\mathbf{s c}^{\mathrm{vor}}$ is a pair $(G, A)$, where $G$ is a Gerstenhaber algebra and $A$ is an associative algebra over the commutative ring $G$.

An algebra over the commutative ring $G$ corresponds to a degree 0 map $\lambda: G \otimes A \rightarrow A$ satisfying

$$
\begin{gathered}
\lambda\left(c c^{\prime}, a\right)=\lambda\left(c, \lambda\left(c^{\prime}, a\right)\right)=(-1)^{|c|\left|c^{\prime}\right|} \lambda\left(c^{\prime}, \lambda(c, a)\right), \\
\lambda\left(c, a a^{\prime}\right)=\lambda(c, a) a^{\prime}=(-1)^{|a||c|} a \lambda\left(c, a^{\prime}\right) .
\end{gathered}
$$

As a consequence we have the following. 
Corollary 3.1.2 The operad $\mathbf{s c}^{\mathrm{vor}}$ has a quadratic presentation $\mathcal{F}\left(E_{v}, R_{v}\right)$ where

$$
E_{v}=\left\langle f_{2}, g_{2}, e_{0,2}, e_{1,1}\right\rangle
$$

and $R_{v}$ is the sub-S-module of $\mathcal{F}^{(2)}\left(E_{v}\right)$ generated by the relations

- $R_{\mathcal{G} e r}$, for the Gerstenhaber structure defined by $f_{2}$ and $g_{2}$ and $R_{\mathcal{A} s s}$ for the associativity of $e_{0,2}$;

- $e_{1,1}$ is an action, with

$$
\begin{gathered}
e_{1,1}\left(\mathrm{id} \otimes e_{1,1}\right)=e_{1,1}\left(f_{2} \otimes \mathrm{id}\right), \\
e_{1,1}\left(\mathrm{id} \otimes e_{0,2}\right)=e_{0,2}\left(e_{1,1} \otimes \mathrm{id}\right)=e_{0,2}\left(\mathrm{id} \otimes e_{1,1}\right) \cdot(213) .
\end{gathered}
$$

Lemma 3.1.3 Algebras over the Koszul dual operad (sc $\left.{ }^{\mathrm{vor}}\right)^{!}$of $\mathbf{s c}^{\mathrm{vor}}$ are of the form $(H, A, \rho)$ where $(H,[],, \times)$ is a $\Lambda \mathcal{G e r - a l g e b r a , ~} A$ is an associative algebra, and $\rho: H \otimes A \rightarrow A$ is a map of degree 0 that satisfies the relations

$$
\begin{aligned}
\rho\left(\left[h, h^{\prime}\right], a\right) & =\rho\left(h, \rho\left(h^{\prime}, a\right)\right)-(-1)^{|h|\left|h^{\prime}\right|} \rho\left(h^{\prime}, \rho(h, a)\right), \\
\rho\left(h, a \cdot a^{\prime}\right) & =\rho(h, a) \cdot a^{\prime}+(-1)^{|a||h|} a \cdot \rho\left(h, a^{\prime}\right), \\
\rho\left(h \times h^{\prime}, a\right) & =0 .
\end{aligned}
$$

Note that the first two equations indicate that the map induced by $\rho$ from $H$ to $\operatorname{End}(A)$ has values in $\operatorname{Der}(A)$ and is a morphism of Lie algebras.

Proof Because $\mathbf{s c}^{\mathrm{vor}}$ has a binary quadratic presentation, we can use the direct computation of its Koszul dual operad presented in Section 2.2.6. Let us denote by $\left(\mathfrak{l}_{2}, \mathfrak{c}_{2}, \mathfrak{n}_{0,2}, \mathfrak{n}_{1,1}\right)$ the dual basis of $\left(f_{2}, g_{2}, e_{0,2}, e_{1,1}\right)$ in $E_{v}^{\vee}$. The degree of $\mathfrak{c}_{2}$ is -1 and all the other elements have degree 0 .

The Koszul dual operad of $\mathbf{s c}^{\mathrm{vor}}$ is $\left(\mathbf{s c}^{\mathrm{vor}}\right)^{!}=\mathcal{F}\left(E_{v}^{\vee}\right) /\left(R_{v}^{\perp}\right)$. The pairing between $E_{v}$ and $E_{v}^{\vee}$ induces a pairing between $\mathcal{F}^{(2)}\left(E_{v}\right)$ and $\mathcal{F}^{(2)}\left(E_{v}^{\vee}\right)$. One gets $R_{v}^{\perp}(c, c, c ; c)$ is the ideal defining $\mathcal{G} e r^{!}$, that is $R_{\Lambda \mathcal{G e r}}$. Similarly $R_{v}^{\perp}(o, o, o ; o)$ is the orthogonal of the associativity relation for $e_{0,2}$, that is, the associativity relation for $\mathfrak{n}_{0,2}$.

The space $\mathcal{F}\left(E_{v}\right)(c, c, o ; o)_{0}$ has dimension 3 and $R_{v}(c, c, o ; o)_{0}$ has dimension 1 . As a consequence, the dimension of $R_{v}^{\perp}(c, c, o ; o)_{0}$ is 2 and corresponds to the first relation.

The space $\mathcal{F}\left(E_{v}\right)(c, o, o ; o)$ has dimension 6 and $R_{v}(c, o, o ; o)$ has dimension 2. Hence the dimension of $R_{v}^{\perp}(c, o, o ; o)$ is 4 and corresponds to the second relation.

The space $\mathcal{F}\left(E_{v}\right)(c, c, o ; o)_{1}$ has dimension 1 and $R_{v}(c, c, o ; o)_{1}$ has dimension 0 . As a consequence, the dimension of $R_{v}^{\perp}(c, c, o ; o)_{-1}$ is 1 and corresponds to the third relation. 
In terms of generators and relations, it expresses as the following.

Corollary 3.1.4 The operad (sc $\left.\mathbf{s}^{\mathrm{vor}}\right)^{!}$has a binary quadratic presentation $\mathcal{F}\left(E_{v^{!}}, R_{v^{!}}\right)$, where

$$
E_{v} !=\left\langle\mathfrak{l}_{2}, \mathfrak{c}_{2}, \mathfrak{n}_{0,2}, \mathfrak{n}_{1,1}\right\rangle
$$

and $R_{v}$ is the sub-S-module of $\mathcal{F}^{(2)}\left(E_{v}\right.$ ) generated by the relations

- $\quad R_{\Lambda \mathcal{G e r}}$, for the $\Lambda \mathcal{G e r - s t r u c t u r e ~ d e f i n e d ~ b y ~} \mathfrak{l}_{2}$ and $\mathfrak{c}_{2}$ and $R_{\mathcal{A s s}}$ for the associativity of $\mathfrak{n}_{0,2}$;

- relations for $\mathfrak{n}_{1,1}$ are

$$
\begin{gathered}
\mathfrak{n}_{1,1}\left(\mathfrak{l}_{2} \otimes \mathrm{id}\right)=\mathfrak{n}_{1,1}\left(\mathrm{id} \otimes \mathfrak{n}_{1,1}\right) \cdot(\mathrm{id}-(213)), \\
\mathfrak{n}_{1,1}\left(\mathrm{id} \otimes \mathfrak{n}_{0,2}\right)=\mathfrak{n}_{0,2}\left(\mathfrak{n}_{1,1} \otimes \mathrm{id}\right)+\mathfrak{n}_{0,2}\left(\mathrm{id} \otimes \mathfrak{n}_{1,1}\right) \cdot(213), \\
\mathfrak{n}_{1,1}\left(\mathfrak{c}_{2} \otimes \mathrm{id}\right)=0 .
\end{gathered}
$$

Theorem 3.1.5 The operad $\mathbf{s c}^{\mathrm{vor}}$ is Koszul.

Proof In order to prove that $\mathbf{s c} \mathbf{c}^{\text {vor }}$ is Koszul, we prove that $\left(\mathbf{s c}^{\mathrm{vor}}\right)^{!}$is Koszul, using the rewriting method explained in [15], and using a part of the computation made by Alm in [1, AppendixA]. Recall that an algebra over $\left(\mathbf{s c}^{\mathrm{vor}}\right)^{!}$is given by the following data.

- A $\Lambda \mathcal{G e r - a l g e b r a ~} H$. We denote by $\left[x_{1}, x_{2}\right]$ the degree 0 bracket and by $x_{1} \times x_{2}$ the degree -1 product.

- An associative algebra $A$. We denote by $a_{1} \cdot a_{2}$ the degree 0 product.

- A map $\rho: H \otimes A \rightarrow A$. We denote by $x \bullet a$ the element $\rho(x, a)$.

The rewriting rules are

$$
\begin{aligned}
\left(a_{1} \cdot a_{2}\right) \cdot a_{3} & \mapsto a_{1} \cdot\left(a_{2} \cdot a_{3}\right), \\
\left(x_{1} \times x_{2}\right) \times x_{3} & \mapsto-x_{1} \times\left(x_{2} \times x_{3}\right), \\
{\left[\left[x_{1}, x_{2}\right], x_{3}\right] } & \mapsto-\left[\left[x_{2}, x_{3}\right], x_{1}\right]-\left[\left[x_{3}, x_{1}\right], x_{2}\right], \\
{\left[x_{1}, x_{2} \times x_{3}\right] } & \mapsto\left[x_{1}, x_{2}\right] \times x_{3}+x_{2} \times\left[x_{1}, x_{3}\right], \\
x_{1} \bullet\left(a_{1} \cdot a_{2}\right) & \mapsto\left(x_{1} \bullet a_{1}\right) \cdot a_{2}+a_{1} \cdot\left(x_{1} \bullet a_{2}\right), \\
\left(x_{1} \times x_{2}\right) \bullet a & \mapsto 0, \\
{\left[x_{1}, x_{2}\right] \bullet a_{1} } & \mapsto x_{1} \bullet\left(x_{2} \bullet a_{1}\right)-x_{2} \bullet\left(x_{1} \bullet a_{1}\right) .
\end{aligned}
$$

In order to study the confluence of critical monomials, it is enough to study the one involving both $x^{\prime} s$ and $a^{\prime} s$ because the one involving only $a^{\prime} s$ corresponds to the 
computation for the operad $\mathcal{A} s s$, and the one involving only $x^{\prime} s$ corresponds to the computation for the operad $\Lambda \mathcal{G e r}$. We know that a way to prove the Koszulity of these 2 operads is precisely to use the confluence of the critical monomials.

Hence the critical monomials left are $\left(x_{1} \bullet\left(\left(a_{1} \cdot a_{2}\right) \cdot a_{3}\right)\right),\left(\left[x_{1}, x_{2}\right] \bullet\left(a_{1} \cdot a_{2}\right)\right)$, $\left(\left[\left[x_{1}, x_{2}\right], x_{3}\right] \bullet a_{1}\right),\left(\left(x_{1} \times x_{2}\right) \bullet\left(a_{1} \cdot a_{2}\right)\right),\left(\left(\left(x_{1} \times x_{2}\right) \times x_{3}\right) \bullet a\right)$ and $\left(\left[x_{1}, x_{2} \times x_{3}\right] \bullet a\right)$. The first three have been proven to be confluent by J Alm. The fourth critical monomial can be rewritten either as

$$
\begin{aligned}
\left(x_{1} \times x_{2}\right) \bullet\left(a_{1} \cdot a_{2}\right) & \mapsto\left(\left(x_{1} \times x_{2}\right) \bullet a_{1}\right) \cdot a_{2}+a_{1} \cdot\left(\left(x_{1} \times x_{2}\right) \bullet a_{2}\right) \\
& \mapsto 0
\end{aligned}
$$

or $\left(x_{1} \times x_{2}\right) \bullet\left(a_{1} \cdot a_{2}\right) \mapsto 0$. The same is true for the fifth critical monomial.

The critical monomial $\left[x_{1}, x_{2} \times x_{3}\right] \bullet a$ can be rewritten either as

$$
\begin{aligned}
{\left[x_{1}, x_{2} \times x_{3}\right] \bullet a } & \mapsto x_{1} \bullet\left(\left(x_{2} \times x_{3}\right) \bullet a\right)-\left(x_{2} \times x_{3}\right) \bullet\left(x_{1} \bullet a\right) \\
& \mapsto 0
\end{aligned}
$$

or

$$
\begin{aligned}
{\left[x_{1}, x_{2} \times x_{3}\right] \bullet a } & \mapsto\left(\left[x_{1}, x_{2}\right] \times x_{3}\right) \bullet a+\left(x_{2} \times\left[x_{1}, x_{3}\right]\right) \bullet a \\
& \mapsto 0 .
\end{aligned}
$$

Hence, all the critical monomials are confluent and $\left(\mathbf{s c}^{\mathrm{vor}}\right)^{!}$is Koszul. As a consequence $\mathbf{s c}^{\text {vor }}$ is a Koszul operad.

\subsection{The operad sc is Koszul}

In this section we follow closely the article by Imma Galvez-Carrillo, Andy Tonks and Bruno Vallette [5] and our paper [9] in order to prove that the homology operad sc is Koszul. Recall from the computation of F Cohen and A Voronov and from [9] the following.

Proposition 3.2.1 An sc-algebra $(G, A, f)$ is a Gerstenhaber algebra $G$ and an associative algebra $A$ together with a central morphism of associative algebras $f: G \rightarrow A$.

Corollary 3.2.2 The operad sc has a presentation of the form $\mathcal{F}\left(E^{\prime}, R^{\prime}\right)$ where

$$
E^{\prime}=\left\langle f_{2}, g_{2}, e_{0,2}, e_{1,0}\right\rangle
$$

and the space of relations $R^{\prime}$ is the sub-S-module of $\mathcal{F}^{(2)}(E) \oplus \mathcal{F}^{(3)}(E)$ defined by the relations 
- $\quad R_{\mathcal{G} e r}$ for the Gerstenhaber structure induced by $f_{2}$ and $g_{2}$ and $R_{\mathcal{A s s}}$ for the associativity of $e_{0,2}$;

- centrality of $e_{1,0}: e_{0,2}\left(e_{1,0} \otimes \mathrm{id}\right)=e_{0,2}\left(\mathrm{id} \otimes e_{1,0}\right) \cdot(21)$;

- a quadratic-cubical relation: $e_{1,0}\left(f_{2}\right)=e_{0,2}\left(e_{1,0} \otimes e_{1,0}\right)$.

This corollary shows clearly that this presentation is quadratic and cubic. In order to apply the theory of [5], one needs a presentation which is quadratic and linear. However, we will see in Proposition 4.2.1 that the quadratic operad $\mathcal{F}\left(E^{\prime}\right) /\left(q R^{\prime}\right)$ obtained by killing the cubical elements in the relations of $R^{\prime}$ plays also an important role for the study of sc.

The idea to obtain a presentation with quadratic-linear relations of $\mathbf{s c}$ is to add a new generator, in order to replace the quadratic-cubical relation by quadratic-linear relations. This new generator $e_{1,1}$, will correspond at the level of algebras to the operation $\lambda(c, a):=f(c) a$. Consequently, we introduce new relations in the operad corresponding to the relations $f(c) a=a f(c)=\lambda(c, a)$ and $\lambda\left(c, f\left(c^{\prime}\right)\right)=f\left(c c^{\prime}\right)=f(c) f\left(c^{\prime}\right)$, that are present in the algebra setting.

Recall the theory explained in [5] for quadratic-linear operads. A quadratic-linear operad is of the form $\mathcal{F}(E) /(R)$ with $R \subset \mathcal{F}^{(1)}(E) \oplus \mathcal{F}^{(2)}(E)$. Such an $R$ is called quadratic-linear. We also ask the presentation to satisfy

(q11) $R \cap E=\{0\}$,

(q12) $(R \otimes E+E \otimes R) \cap \mathcal{F}^{(2)}(E) \subset R \cap \mathcal{F}^{(2)}(E)$.

Proposition 3.2.3 The operad sc has a presentation $\mathcal{F}(E, R)$, where

$$
E=\left\langle f_{2}, g_{2}, e_{0,2}, e_{1,1}, e_{1,0}\right\rangle
$$

and the space of relations $R$ is the sub-S-module of $\mathcal{F}^{(1)}(E) \oplus \mathcal{F}^{(2)}(E)$ defined by $R=R_{v} \oplus R\left(e_{1,0}\right)$, where $R_{v}$ is the space of quadratic relations of $\mathbf{s c}^{\mathrm{vor}}$ and $R\left(e_{1,0}\right)$ is the sub-S-module of $\mathcal{F}(E)$ generated by the following relations:

- two quadratic-linear relations: $e_{1,1}=e_{0,2}\left(e_{1,0} \otimes \mathrm{id}\right)$ and $e_{1,1}=e_{0,2}\left(\mathrm{id} \otimes e_{1,0}\right)$. (21),

- a new quadratic relation: $e_{1,1}\left(\mathrm{id} \otimes e_{1,0}\right)=e_{1,0}\left(f_{2}\right)$.

Moreover this presentation satisfies (q11) and (q12).

Here we recall the definition of a Koszul quadratic-linear operad given in [5]. 
Definition 3.2.4 Let $q$ denote the projection $\mathcal{F}(E) \rightarrow \mathcal{F}^{(2)}(E)$ and let $q R$ be the image of $R$ under this projection. A quadratic-linear operad $\mathcal{P}=\mathcal{F}(E) /(R)$ satisfying (q11) and (q12) is said to be $K o s z u l$ if $q \mathcal{P}:=\mathcal{F}(E) /(q R)$ is a quadratic Koszul operad. Its Koszul dual cooperad is $(\mathcal{P})^{i}=\left((q \mathcal{P})^{i}, \partial_{\varphi}\right)$ where the differential $\partial_{\varphi}$ depends on the quadratic-linear relations.

In the case of sc presented as in Proposition 3.2.3, the projection of $R=R_{v} \oplus R\left(e_{1,0}\right)$ onto $\mathcal{F}^{(2)}(E)$ is $q R=R_{v} \oplus q R\left(e_{1,0}\right)$, where $q R\left(e_{1,0}\right)$ is the sub- $\mathbb{S}$-module of $\mathcal{F}^{(2)}(E)$ generated by the relations $0=e_{0,2}\left(e_{1,0} \otimes \mathrm{id}\right), 0=e_{0,2}\left(e_{1,0} \otimes \mathrm{id}\right)(12)$ and $e_{1,1}\left(\mathrm{id} \otimes e_{1,0}\right)=e_{1,0}\left(f_{2}\right)$.

Consequently a $q \mathbf{s c}$-algebra is an $\mathbf{s c}^{\text {vor }}$-algebra $(G, A, \lambda)$ endowed with a degree 0 linear map $f: G \rightarrow A$ satisfying $f(c) a=a f(c)=0$ and $\lambda\left(c, f\left(c^{\prime}\right)\right)=f\left(c c^{\prime}\right)$ for all $c, c^{\prime} \in G, a \in A$. As in [9], the operad $q \mathbf{s c}$ is obtained as the result of a distributive law between the operad $\mathbf{s c}^{\mathrm{vor}}$ and $\mathcal{F}\left(e_{1,0}\right)$. The distributive law is given by

$$
\begin{aligned}
\mathbf{s c}^{\mathrm{vor}} \circ \mathcal{F}\left(e_{1,0}\right) & \rightarrow \mathcal{F}\left(e_{1,0}\right) \circ \mathbf{s c}^{\mathrm{vor}}, \\
e_{0,2}\left(e_{1,0} \otimes \mathrm{id}\right), e_{0,2}\left(e_{1,0} \otimes \mathrm{id}\right)(12) & \mapsto 0, \\
e_{1,1}\left(\mathrm{id} \otimes e_{1,0}\right) & \mapsto e_{1,0}\left(f_{2}\right) .
\end{aligned}
$$

Proposition 3.2.5 The operad $q \mathbf{s c}$ is identical to the operad $\mathcal{F}\left(e_{1,0}\right) \circ \mathbf{s c}^{\mathrm{vor}}$, with composition given by the distributive law (3).

Theorem 3.2.6 The operad $q \mathbf{s c}$ is a quadratic Koszul operad. As a consequence, there exists a quadratic-linear presentation of the operad sc so that sc is a quadratic-linear Koszul operad.

Proof From [15, Chapter8], one has that $q \mathbf{s c}=\mathcal{F}\left(e_{1,0}\right) \circ \mathbf{s c}^{\mathrm{vor}}$ is Koszul since $\mathbf{s c}^{\mathrm{vor}}$ and $\mathcal{F}\left(e_{1,0}\right)$ are Koszul colored operads. By definition, it means that sc is a quadraticlinear Koszul operad.

\subsection{Description of the Koszul dual operad (sc)! of sc.}

In Proposition 3.2.5, we have described $q \mathbf{s c}$ as a distributive law between $\mathbf{s c}^{\mathrm{vor}}$ and $\mathcal{F}\left(e_{1,0}\right)$. As a consequence $(q \mathbf{s c})^{!}=\left(\mathbf{s c}^{\mathrm{vor}}\right)^{!} \circ \mathcal{F}\left(e_{1,0}\right)^{!}$, with the operad structure given by the signed dual of the distributive law (3). Recall from Corollary 3.1.4 that $\left\{\mathfrak{l}_{2}, \mathfrak{c}_{2}, \mathfrak{n}_{0,2}, \mathfrak{n}_{1,1}\right\}$ is the dual basis of $\left\{f_{2}, g_{2}, e_{2,0}, e_{1,1}\right\}$ that generates $E_{v^{!}}$. From relation (1), one has $\mathcal{F}\left(e_{1,0}\right)^{!}=\mathcal{F}\left(\mathfrak{n}_{1,0}\right)$ where $\mathfrak{n}_{1,0}$ has degree -1 . The dual of the 
distributive law is given by

$$
\begin{aligned}
\mathcal{F}\left(\mathfrak{n}_{1,0}\right) \circ\left(\mathbf{s c}^{\mathrm{vor}}\right)^{!} & \rightarrow\left(\mathbf{s c}^{\mathrm{vor}}\right)^{!} \circ \mathcal{F}\left(\mathfrak{n}_{1,0}\right), \\
\mathfrak{n}_{1,0}\left(\mathfrak{l}_{2}\right) & \mapsto \mathfrak{n}_{1,1}\left(\mathrm{id} \otimes \mathfrak{n}_{1,0}\right) \cdot(\mathrm{id}-(21)), \\
\mathfrak{n}_{1,0}\left(\mathfrak{c}_{2}\right) & \mapsto 0 .
\end{aligned}
$$

Consequently, a $(q \mathbf{s c})^{!}$-algebra is an $\left(\mathbf{s c}^{\mathrm{vor}}\right)^{!}$-algebra $(H, A, \rho)$ satisfying conditions of Lemma 3.1.3, together with a linear map $\beta: H \rightarrow A$ of degree -1 satisfying

$$
\begin{aligned}
& \beta\left(\left[h, h^{\prime}\right]\right)=(-1)^{|h|} \rho\left(h, \beta\left(h^{\prime}\right)\right)-(-1)^{|h|\left|h^{\prime}\right|+\left|h^{\prime}\right|} \rho\left(h^{\prime}, \beta(h)\right), \\
& \beta\left(h \times h^{\prime}\right)=0 .
\end{aligned}
$$

In order to understand the structure of an (sc)!-algebra it is then enough to understand the differential on the operad $(q \mathbf{s c})^{!}$that comes from the nonquadraticity of the operad sc.

Let $\varphi: q R \rightarrow E$ be defined by

$$
\begin{aligned}
\varphi\left(e_{0,2}\left(e_{1,0} \otimes \mathrm{id}\right)\right) & =\varphi\left(e_{0,2}\left(\mathrm{id} \otimes e_{1,0}\right) \cdot(21)\right)=e_{1,1}, \\
\varphi\left(R_{v}\right) & =0, \\
\varphi\left(e_{1,1}\left(\mathrm{id} \otimes e_{1,0}\right)-e_{1,0}\left(f_{2}\right)\right) & =0 .
\end{aligned}
$$

The Koszul dual cooperad of $q \mathbf{s c}$ is $(q \mathbf{s c})^{i}=C\left(s E, s^{2} q R\right)$, with the notation of Section 2.2.6. To $\varphi$ is associated the composite map

$$
(q \mathbf{s c})^{i} \rightarrow s^{2} q R \stackrel{s^{-1} \varphi}{\longrightarrow} s E .
$$

There exists a unique coderivation $\tilde{\partial}_{\varphi}:(q \mathbf{s c})^{i} \rightarrow \mathcal{F}^{c}(s E)$ which extends this map. Moreover, $\widetilde{\partial}_{\varphi}$ induces a square zero coderivation $\partial_{\varphi}$ on the Koszul dual cooperad $(q \mathbf{s c})^{i}$. The Koszul dual cooperad of $\mathbf{s c}$ is by definition $\mathbf{s c}^{i}=\left(C\left(s E, s^{2} q R\right), \partial_{\varphi}\right)$.

Recall from (1) that $(q \mathbf{s c})^{!}=(\Lambda(q \mathbf{s c i}))^{*}$. As a consequence, $\mathbf{s c}^{!}=\left((q \mathbf{s c})^{!}, d_{\varphi}\right)$, where $d_{\varphi}$ is obtained as a combination of transpose and signed suspension of $\partial_{\varphi}$. Namely, $\mathbf{s c}^{!}$is a differential graded operad and we have the following Proposition.

Proposition 3.3.1 An algebra over sc! consists in a $d g \Lambda \mathcal{G}$ er-algebra $\left(H,[],, \times, d_{H}\right)$, a $d g$ associative algebra $\left(A, d_{A}\right)$, an action $\rho: H \otimes A \rightarrow A$ and a degree -1 map $\beta: H \rightarrow A$ such that, for all $h \in H, a \in A$, we have $d_{A}(\beta(h))=-\beta\left(d_{H} h\right)$ and that the relations (2) and (4) are satisfied. Moreover, the following relation is satisfied:

$$
d_{A} \rho(h, a)=\rho\left(d_{H} h, a\right)+(-1)^{|h|} \rho\left(h, d_{A} a\right)+\beta(h) a-(-1)^{|a|(|h|+1)} a \beta(h) .
$$


Note that relation (5) says that the map $\beta: H \rightarrow A$ is central up to homotopy having the map $\rho: H \otimes A \rightarrow A$ as the homotopy operator. For a geometrical description of the above relations in terms of the Kontsevich compactification [14], we refer the reader to the first author [8], Kajiura and Stasheff [11] and the authors [9].

Remark 3.3.2 There is a more compact way to understand what are sc'-algebras. Let $\left(A, d_{A}\right)$ be a dg associative algebra. Let $\operatorname{Der}(A)$ be the $\mathrm{dg}$ Lie algebra of derivations of $A$. For a given $a \in A$ we denote by $D_{a}$ the inner derivation which is defined by $D_{a}(x)=a x-(-1)^{|a||x|} x a$. The graded $k$-vector space $s A$ is a module over $\operatorname{Der}(A)$ via the action $[d, s a]=(-1)^{|d|} s d(a)$. Consequently, there is a structure of graded Lie algebra on $\operatorname{Der}_{+}(A)=\operatorname{Der}(A) \oplus s A$. A short computation shows that the differential $\partial_{+}(d+s a)=\partial d+D_{a}-s d_{A}(a)$ endows $\operatorname{Der}_{+}(A)$ with a structure of dg Lie algebra. Furthermore, any dg Lie algebra is a dg $\Lambda \mathcal{G}$ er-algebra, setting the product to be 0 .

As a consequence, one has the following.

An algebra over sc! consists in a $d g \Lambda \mathcal{G e r - a l g e b r a ~}\left(H,[],, \times, d_{H}\right)$, a dg associative algebra $\left(A, d_{A}\right)$, and a morphism of $d g \Lambda \mathcal{G e r - a l g e b r a s} \gamma: H \rightarrow \operatorname{Der}_{+}(A)$.

Translating the proposition in the language of operads, one gets the following corollary.

Corollary 3.3.3 The differential graded operad $(\mathbf{s c})^{!}$has a presentation $\mathcal{F}\left(E_{!}, R_{!}\right)$, where

$$
E_{!}=\left\langle\mathfrak{l}_{2}, \mathfrak{c}_{2}, \mathfrak{n}_{0,2}, \mathfrak{n}_{1,1}, \mathfrak{n}_{1,0}\right\rangle
$$

and the vector space $R_{!}$is the sub-S-module of $\mathcal{F}^{(2)}\left(E_{!}\right)$generated by the relations:

- $\quad R_{\Lambda \mathcal{G e r}}$, for the $\Lambda \mathcal{G}$ er-structure defined by $\mathfrak{l}_{2}$ and $\mathfrak{c}_{2}$ and $R_{\mathcal{A} s s}$ for the associativity of $\mathfrak{n}_{0,2}$;

- relations for $\mathfrak{n}_{1,1}$ are

$$
\begin{gathered}
\mathfrak{n}_{1,1}\left(\mathfrak{l}_{2} \otimes \mathrm{id}\right)=\mathfrak{n}_{1,1}\left(\mathrm{id} \otimes \mathfrak{n}_{1,1}\right) \cdot(\mathrm{id}-(213)), \\
\mathfrak{n}_{1,1}\left(\mathrm{id} \otimes \mathfrak{n}_{0,2}\right)=\mathfrak{n}_{0,2}\left(\mathfrak{n}_{1,1} \otimes \mathrm{id}\right)+\mathfrak{n}_{0,2}\left(\mathrm{id} \otimes \mathfrak{n}_{1,1}\right) \cdot(213), \\
\mathfrak{n}_{1,1}\left(\mathfrak{c}_{2} \otimes \mathrm{id}\right)=0 ;
\end{gathered}
$$

- relations for $\mathfrak{n}_{1,0}$ are

$$
\begin{gathered}
\mathfrak{n}_{1,0}\left(\mathfrak{l}_{2}\right)=\mathfrak{n}_{1,1}\left(\mathrm{id} \otimes \mathfrak{n}_{1,0}\right) \cdot((12)-(21)), \\
\mathfrak{n}_{1,0}\left(\mathfrak{c}_{2}\right)=0 .
\end{gathered}
$$

The differential is given by $d \mathfrak{n}_{1,1}=\mathfrak{n}_{0,2}\left(\mathfrak{n}_{1,0} \otimes \mathrm{id}\right)-\mathfrak{n}_{0,2}\left(\mathrm{id} \otimes \mathfrak{n}_{1,0}\right) \cdot(21)$ and vanishes elsewhere. 


\subsection{On the homology of sc!}

In [9], we have considered the $0^{\text {th }}$ homology operad of $\mathcal{S C}$. In particular, the description of $H_{0}(\mathcal{S C})^{!}([9$, Proposition 6.3.2]) is the following.

Proposition 3.4.1 The differential graded operad $H_{0}(\mathcal{S C})^{!}$has a presentation given by $\mathcal{F}\left(E_{0}, R_{0}\right)$, where

$$
E_{0}=\left\langle\mathfrak{l}_{2}, \mathfrak{n}_{0,2}, \mathfrak{n}_{1,1}, \mathfrak{n}_{1,0}\right\rangle
$$

and the vector space $R_{0}$ is the sub-S-module of $\mathcal{F}^{(2)}\left(E_{0}\right)$ generated by the relations:

- $\quad R_{\mathcal{L} i e}$, for the $\mathcal{L} i e-s t r u c t u r e$ defined by $\mathfrak{l}_{2}$ and $R_{\mathcal{A} s s}$ for the associativity of $\mathfrak{n}_{0,2}$;

- relations for $\mathfrak{n}_{1,1}$ are

$$
\begin{gathered}
\mathfrak{n}_{1,1}\left(\mathfrak{l}_{2} \otimes \mathrm{id}\right)=\mathfrak{n}_{1,1}\left(\mathrm{id} \otimes \mathfrak{n}_{1,1}\right) \cdot(\mathrm{id}-(213)), \\
\mathfrak{n}_{1,1}\left(\mathrm{id} \otimes \mathfrak{n}_{0,2}\right)=\mathfrak{n}_{0,2}\left(\mathfrak{n}_{1,1} \otimes \mathrm{id}\right)+\mathfrak{n}_{0,2}\left(\mathrm{id} \otimes \mathfrak{n}_{1,1}\right) \cdot(213) ;
\end{gathered}
$$

- relations for $\mathfrak{n}_{1,0}$ are

$$
\mathfrak{n}_{1,0}\left(\mathfrak{l}_{2}\right)=\mathfrak{n}_{1,1}\left(\mathrm{id} \otimes \mathfrak{n}_{1,0}\right) \cdot((12)-(21)) .
$$

The differential is given by $d \mathfrak{n}_{1,1}=\mathfrak{n}_{0,2}\left(\mathfrak{n}_{1,0} \otimes \mathrm{id}\right)-\mathfrak{n}_{0,2}\left(\mathrm{id} \otimes \mathfrak{n}_{1,0}\right) \cdot(21)$ and is zero on all the other generators.

From this, it is easy to prove the following corollary.

Corollary 3.4.2 The dg operad sc! is the operad composite $\Lambda \mathcal{C}$ om $\circ H_{0}(\mathcal{S C})^{!}$together with the distributive law given by

$$
\begin{aligned}
H_{0}(\mathcal{S C}) ! & \circ \Lambda \mathcal{C} \text { om } \\
\mathfrak{l}_{2}\left(\mathrm{id} \otimes \mathfrak{c}_{2}\right) & \mapsto \mathfrak{c}_{2}\left(\mathfrak{l}_{2} \otimes \mathrm{id}\right)+\mathfrak{c}_{2}\left(\mathrm{id} \otimes \mathfrak{l}_{2}\right) \cdot(213), \\
\mathfrak{n}_{1,1}\left(\mathfrak{c}_{2} \otimes \mathrm{id}\right) & \mapsto 0, \\
\mathfrak{n}_{1,0}\left(\mathfrak{c}_{2}\right) & \mapsto 0 .
\end{aligned}
$$

As a consequence we get the following.

Theorem 3.4.3 Algebras over the homology of the operad sc! are triples $(H, A, \beta)$ where $H$ is a $\Lambda \mathcal{G}$ er-algebra, $A$ is an associative algebra and $\beta: H \rightarrow A$ is a central map of degree -1 satisfying $\beta(x \times y)=0$. 
Proof Recall from [9, Theorem 7.2.5] that algebras over the homology of the operad $H_{0}(\mathcal{S C})^{!}$are triples $(L, A, f)$ where $L$ is a Lie algebra, $A$ is an associative algebra and $f: L \rightarrow A$ is a central map of degree -1 . Using the Künneth formula for the plethysm product $\circ$ of $\mathbb{S}$-modules, as in Fresse [4, Lemma 2.1.3], we obtain that $H_{*}\left(\mathbf{s c}^{!}\right)=\Lambda \mathcal{C}$ om $\circ H_{*}\left(H_{0}(\mathcal{S C})^{!}\right)$with the distributive law given by

$$
\begin{aligned}
H_{*}\left(H_{0}(\mathcal{S C})^{!}\right) \circ \Lambda \mathcal{C o m} & \rightarrow \Lambda \mathcal{C o m} \circ H_{*}\left(H_{0}(\mathcal{S C})^{!}\right), \\
{\left[\mathfrak{l}_{2}\right]\left(\mathrm{id} \otimes \mathfrak{c}_{2}\right) } & \mapsto \mathfrak{c}_{2}\left(\left[\mathfrak{l}_{2}\right] \otimes \mathrm{id}\right)+\mathfrak{c}_{2}\left(\mathrm{id} \otimes\left[\mathfrak{l}_{2}\right]\right) \cdot(213), \\
{\left[\mathfrak{n}_{1,0}\right]\left(\mathfrak{c}_{2}\right) } & \mapsto 0,
\end{aligned}
$$

where $[x]$ denotes the image of a cycle $x$ in $H_{*}\left(H_{0}(\mathcal{S C})^{!}\right)$.

\section{On the spectral sequence}

In this section we will show that the spectral sequence $E(\mathcal{S C})$ associated to the stratification of the compactification of points in the upper half plane collapses at the second stage. We prove that, as an $\mathbb{S}$-module, $E^{2}(\mathcal{S C})$ corresponds to the $\mathbb{S}$-module defined by $\mathbf{s c}$, but prove that the operad structures are not isomorphic.

\subsection{On the first sheet of the spectral sequence}

For this section, we refer to $[17 ; 8 ; 2]$.

For the compactification of points we are considering two different spaces: the space $C(n)$ of configurations of $n \geqslant 2$ points in the disk modded out by the action of the group of dilatations and translations, of dimension $2 n-3$; the space $C(n, m)$, with $2 n+m \geqslant 2$, of configurations of $n$ points in the upper half plane, $m$ points on the line, modded out by the action of the group of dilatations and translations along the line, of dimension $2 n+m-2$.

The operad $\mathcal{S C}$ is homotopy equivalent to the Fulton-MacPherson compactification of $C(n)$ for $\mathcal{S C}(n, 0 ; c)$ and of $C(n, m)$ for $\mathcal{S C}(n, m ; o)$. Since $C(1)$ and $C(0,1)$ are not well defined, we introduce both $\mathcal{S C}(1,0 ; c)$ and $\mathcal{S C}(0,1 ; o)$ as the one point spaces containing the identity element of the closed and open colors, respectively. It has been proven by Getzler and Jones in [6], that the filtration associated to the stratification of the compactification of $C(n)$ induces a spectral sequence $E\left(\mathcal{D}_{2}\right)$, whose first sheet coincides with the cobar construction of the cooperad $(\mathcal{G e r})^{i}$. Furthermore, the spectral sequence collapses at the second stage, and $E^{2}\left(\mathcal{D}_{2}\right)$ coincides, as an operad, with $\mathcal{G} e r$. We will then focus on the open part of the Swiss-cheese operad. From [8, Theorem 5.2], 
there is a stratification of $\overline{C(n, m)}$ indexed by partially planar trees, which induces a topological filtration

$$
F^{p}:=F^{p}(\bar{C})=\{\text { closure of the union of strata of dimension } p\} .
$$

It yields a spectral sequence, whose first sheet is given by

$$
E^{1}(\mathcal{S C})_{p, q}=H_{p+q}\left(F^{p}, F^{p-1}\right)=H^{-q}\left(F^{p} \backslash F^{p-1}\right) .
$$

Let $\mathbb{T}(n, m)_{p}$ be the set of partially planar trees with $n$ closed inputs, $m$ open inputs, the output being open and $v:=2 n+m-p-1$ vertices. To any vertex $v_{i}$ of a tree $T \in \mathbb{T}(n, m)_{p}$ is associated the triple $\left(n_{i}, m_{i}, x_{i}\right)$ corresponding respectively, to its closed, open inputs, and its output. One has the relation

$$
\begin{aligned}
& \sum_{i=1}^{v} n_{i}=n+\sum_{i=1}^{v} \delta_{x_{i}, c} \\
& \sum_{i=1}^{v} m_{i}=m+\sum_{i=1}^{v} \delta_{x_{i}, o}-1 .
\end{aligned}
$$

Since any tree $T \in \mathbb{T}(n, m)_{p}$ is responsible for a strata $C_{T}:=\prod_{i=1}^{v} \mathcal{S C}\left(n_{i}, m_{i} ; x_{i}\right)$ one gets that

$$
E^{1}(\mathcal{S C})(n, m)_{p, q}=\bigoplus_{\substack{T \in \mathbb{T}(n, m)_{p}, u_{1}+\cdots+u_{v}=-q}} \bigotimes_{i=1}^{v} H^{u_{i}}\left(\mathcal{S C}\left(n_{i}, m_{i} ; x_{i}\right)\right)
$$

Because we are not exactly using the notation of [2], we need the following Lemma.

Lemma 4.1.1 The operad $E^{1}(\mathcal{S C})$ coincides with the cobar construction of the coop$\operatorname{erad}\left(\Lambda_{c} \Lambda \mathbf{s c}\right)^{*}$. More precisely, one has

$$
E^{1}(\mathcal{S C})(n, m)_{p, q}=\Omega\left(\left(\Lambda_{c} \Lambda \mathbf{s c}\right)^{*}\right)(n, m ; o)_{p+q}^{(2 n+m-p-1)},
$$

where the upper index corresponds to the weight grading by the number of vertices of the trees involved and the lower index corresponds to the total degree.

Proof Recall that for any cooperad $\mathcal{C}, \Omega(\mathcal{C})$ is the free operad $\mathcal{F}\left(s^{-1} \overline{\mathcal{C}}\right)$, where $\overline{\mathcal{C}}$ is the coaugmentation ideal of the cooperad. Since $E^{1}(\mathcal{S C})$ is also a free 2-colored 
operad, they have the same description in terms of trees. One has

$$
\begin{aligned}
H^{u}(\mathcal{S C}(n, m ; x)) & =\mathbf{s c}^{*}(n, m ; x)_{-u} \\
& =\left(\Lambda^{-1} \mathbf{s c}^{*}\right)(n, m ; x)_{-u+n+m-1} \\
& =\left(\Lambda_{c}^{-1} \Lambda^{-1} \mathbf{s c}^{*}\right)(n, m ; x)_{-u+2 n+m-1-\delta_{x, c}} \\
& =\left(s^{-1} \Lambda_{c}^{-1} \Lambda^{-1} \mathbf{s c}^{*}\right)(n, m ; x)_{-u+2 n+m-1-\delta_{x, c}-1} .
\end{aligned}
$$

Using the description of $E^{1}(\mathcal{S C})$ in (7) and the formulas in (6), one gets

$$
\begin{aligned}
\sum_{i=1}^{v}\left(-u_{i}+2 n_{i}+m_{i}-1-\delta_{x_{i}, c}-1\right) & =q+2 n+m+\sum_{i=1}^{v} \delta_{x_{i}, c}+\delta_{x_{i}, o}-2 v-1 \\
& =q+2 n+m-v-1=q+p,
\end{aligned}
$$

which explains the grading obtained. From [8; 2], we know the differentials of the two operads coincide. As a consequence, the two differential graded operads coincide.

\subsection{On the second sheet of the spectral sequence}

Theorem 3.2.6 asserts that sc is a Koszul operad, which expresses that

$$
\Omega\left(\mathbf{s c}^{i}\right) \rightarrow \mathbf{s c}
$$

is a quasi-isomorphism of operads. Since all the graded vector spaces involved are finite dimensional, there is a quasi-isomorphism of cooperads

$$
\mathbf{s c}^{*} \rightarrow\left(\Omega\left(\mathbf{s c}^{i}\right)\right)^{*}=B\left(\left(\mathbf{s c}^{i}\right)^{*}\right) \stackrel{(1)}{=} B\left(\Lambda\left(\mathbf{s c}^{!}\right)\right) .
$$

Applying the bar-cobar adjunction we have a sequence of quasi-isomorphisms,

$$
\Omega\left(\mathbf{s c}^{*}\right) \rightarrow \Omega B\left(\Lambda\left(\mathbf{s c}^{!}\right)\right) \rightarrow \Lambda\left(\mathbf{s c}^{!}\right) .
$$

Now applying the functor $\Lambda_{c}^{-1} \Lambda^{-1}$ to the above morphism and using Lemma 4.1.1, we finally have the quasi-isomorphism

$$
E^{1}(\mathcal{S C})=\Omega\left(\left(\Lambda_{c} \Lambda \mathbf{s c}\right)^{*}\right) \rightarrow \Lambda_{c}^{-1}\left(\mathbf{s c}^{!}\right) .
$$

Proposition 4.2.1 The operad $E^{2}(\mathcal{S C})$ is the quadratic operad $\mathcal{F}\left(E^{\prime}, q R^{\prime}\right)$, where

$$
E^{\prime}=\left\langle f_{2}, g_{2}, e_{0,2}, e_{1,0}\right\rangle
$$

and the space of relations $q R^{\prime}$ is the sub-S-module of $\mathcal{F}^{(2)}\left(E^{\prime}\right)$ defined by the relations

- $R_{\mathcal{G} e r}$ for the Gerstenhaber structure induced by $f_{2}$ and $g_{2}$ and $R_{\mathcal{A} s s}$ for the associativity of $e_{0,2}$; 
- centrality of $e_{1,0}: e_{0,2}\left(e_{1,0} \otimes \mathrm{id}\right)=e_{0,2}\left(\mathrm{id} \otimes e_{1,0}\right) \cdot(21)$;

- the quadratic relation: $e_{1,0}\left(f_{2}\right)=0$.

Equivalently, algebras over the operad $E^{2}(\mathcal{S C})$ are triples $(G, A, f)$ where $G$ is a Gerstenhaber algebra, $A$ is an associative algebra, $f: G \rightarrow A$ is a central degree 0 map satisfying $f\left(g g^{\prime}\right)=0$, for all $g, g^{\prime} \in G$.

Proof The operad $E^{2}(\mathcal{S C})$ is the homology of the dg operad $E^{1}(\mathcal{S C})$. Due to the quasi-isomorphism (8), it is the homology of the operad $\Lambda_{c}^{-1}\left(\mathbf{s c}^{\prime}\right)$. From the computation of the homology of $\mathbf{s c}^{!}$obtained in Theorem 3.4.3, we get the result.

Theorem 4.2.2 The spectral sequence $E(\mathcal{S C})$ collapses at the second stage.

Proof Proposition 4.2.1 implies we have that, as an $\mathbb{S}$-module, $E^{2}(\mathcal{S C})(n, m ; o)=$ $\mathcal{G} \operatorname{er}(n) \otimes \mathcal{A s s}(m)=H_{*}(\mathcal{S C})(n, m ; o)$. Because the spectral sequence converges to the homology of $\mathcal{S C}$, and because the dimension of the second sheet is the dimension of the target, one gets that $E(\mathcal{S C})$ collapses at the second stage.

\subsection{Conclusion}

We have shown the following.

Algebras over $H_{*}(S C)$ are triples $(G, A, f)$ where $G$ is a Gerstenhaber algebra, $A$ is an associative algebra and $f: G \rightarrow A$ is a central map such that $f\left(g g^{\prime}\right)=f(g) f\left(g^{\prime}\right)$, whereas algebras over $E^{\infty}(S C)$ are triples $(G, A, f)$, where $G$ is a Gerstenhaber algebra, $A$ is an associative algebra and $f: G \rightarrow A$ is a central map such that $f\left(g g^{\prime}\right)=0$.

Note that the operad $E^{\infty}(\mathcal{S C})=E^{2}(\mathcal{S C})$ obtained is exactly the quadratic operad associated to the quadratic-cubical presentation of the operad sc of Corollary 3.2.2. This is not a surprise because it is the graded operad associated to a filtration of sc. Note also that there is no hope of having a theorem similar to the one obtained by Getzler and Jones in [6] for the little disks operad, that is, an isomorphism between $E^{\infty}(\mathcal{S C})$ and sc. Indeed, one has the following.

Proposition 4.3.1 $E^{2}(\mathcal{S C})$ and sc are not isomorphic.

Proof If they were, there would be a bijective morphism of operads $\varphi: E^{2}(\mathcal{S C}) \rightarrow \mathbf{s c}$. Let $f_{2}, g_{2}, e_{0,2}, e_{1,0}$ denote the generators of $E^{2}(\mathcal{S C})$ and $f_{2}^{\prime}, g_{2}^{\prime}, e_{0,2}^{\prime}, e_{1,0}^{\prime}$ the generators of $\mathbf{s c}$. 
The generators we are concerned with are $f_{2}$ and $e_{1,0}$. Note that $E^{2}(\mathcal{S C})(c, c ; c)_{0}$ is 1 -dimensional so $f_{2}$ is a generator of this $k$-vector space. The same argument holds for the choice of $e_{1,0}, f_{2}^{\prime}$ and $e_{1,0}^{\prime}$. Hence, because of degree and arity reasons, there exist $\lambda, \mu \in k$ such that $\varphi\left(f_{2}\right)=\lambda f_{2}^{\prime}$ and $\varphi\left(e_{1,0}\right)=\mu e_{1,0}^{\prime}$. But we have that $\varphi\left(e_{1,0}\left(f_{2}\right)\right)=\varphi(0)=\lambda \mu e_{1,0}^{\prime}\left(f_{2}^{\prime}\right)$ and $e_{1,0}^{\prime}\left(f_{2}^{\prime}\right) \neq 0 \in \mathbf{s c}(c, c ; o)$. So $\lambda \mu=0$, which contradicts the fact that $\varphi$ is bijective.

\section{References}

[1] J Alm, Two-colored noncommutative Gerstenhaber formality and infinity Duflo isomorphism arXiv:1104.2194

[2] V Dolgushev, Formality theorem for Hochschild cochains via transfer, Lett. Math. Phys. 97 (2011) 109-149 MR2821234

[3] M Flato, M Gerstenhaber, A A Voronov, Cohomology and deformation of Leibniz pairs, Lett. Math. Phys. 34 (1995) 77-90 MR1334037

[4] B Fresse, Koszul duality of operads and homology of partition posets, from: "Homotopy theory: Relations with algebraic geometry, group cohomology, and algebraic $K$-theory", (P Goerss, S Priddy, editors), Contemp. Math. 346, Amer. Math. Soc., Providence, RI (2004) 115-215 MR2066499

[5] I Gálvez-Carrillo, A Tonks, B Vallette, Homotopy Batalin-Vilkovisky algebras, J. Noncommut. Geom. 6 (2012) 539-602 MR2956319

[6] E Getzler, J D S Jones, Operads, homotopy algebra and iterated integrals for double loop spaces arXiv:hep-th/9403055

[7] V Ginzburg, M Kapranov, Koszul duality for operads, Duke Math. J. 76 (1994) 203-272 MR1301191

[8] E Hoefel, OCHA and the swiss-cheese operad, J. Homotopy Relat. Struct. 4 (2009) 123-151 MR2520990 arXiv:0710.3546v5

[9] E Hoefel, M Livernet, Open-closed homotopy algebras and strong homotopy Leibniz pairs through Koszul operad theory, Lett. Math. Phys. 101 (2012) 195-222 MR2947961

[10] H Kajiura, J Stasheff, Homotopy algebras inspired by classical open-closed string field theory, Comm. Math. Phys. 263 (2006) 553-581 MR2211816

[11] H Kajiura, J Stasheff, Open-closed homotopy algebra in mathematical physics, J. Math. Phys. 47 (2006) 023506, 28 MR2208168

[12] H Kajiura, J Stasheff, Homotopy algebras of open-closed strings, from: "Groups, homotopy and configuration spaces", (N Iwase, T Kohno, R Levi, D Tamaki, J Wu, editors), Geom. Topol. Monogr. 13 (2008) 229-259 MR2508208 
[13] M Kontsevich, Operads and motives in deformation quantization, Lett. Math. Phys. 48 (1999) 35-72 MR1718044

[14] M Kontsevich, Deformation quantization of Poisson manifolds, Lett. Math. Phys. 66 (2003) 157-216 MR2062626

[15] J-L Loday, B Vallette, Algebraic operads, Grundlehren der Mathematischen Wissenschaften 346, Springer, Heidelberg (2012) MR2954392

[16] P Salvatore, Configuration spaces with summable labels, from: "Cohomological methods in homotopy theory", (J Aguadé, C Broto, C Casacuberta, editors), Progr. Math. 196, Birkhäuser, Basel (2001) 375-395 MR1851264

[17] A A Voronov, The Swiss-cheese operad, from: "Homotopy invariant algebraic structures”, (J-P Meyer, J Morava, W S Wilson, editors), Contemp. Math. 239, Amer. Math. Soc., Providence, RI (1999) 365-373 MR1718089

Departamento de Matemática, Universidade Federal do Paraná

Universidade Federal do Paraná - C.P. 019081, 81531-990 Curitiba, PR - Brazil

Sorbonne Paris Cité, Université Paris 13

LAGA, CNRS UMR 7539, 99 avenue Jean-Baptiste Clément, F-93430 Villetaneuse, France hoefel@ufpr.br, livernet@math.univ-paris13.fr

Received: 17 October 2012 Revised: 1 February 2013 